\title{
OPTICAL WAVEGUIDE DEVICES FOR BIOANALYSIS
}

\author{
James S Wilkinson \\ University of Southampton, UK
}

\begin{abstract}
Integrated optical waveguides offer great potential as versatile platforms for constructing advanced biosensors, optical cell-sorters and integrated optofluidic systems, exploiting the technological approaches of microelectronics and guided-wave optics to realise low-cost on-chip systems. Progress towards optical integration in microsystems for bioanalysis will be discussed, with examples in key applications, and challenges and opportunities will be described.
\end{abstract}

\section{INTRODUCTION}

Optical waveguides are ideal structures for the integration of advanced optical functions in microsystems. Waveguides are normally formed by deposition and structuring of a thin dielectric film on the surface of a substrate and the waveguide power is confined near the surface yielding a strong controllable interaction. Microfabrication of optical waveguides enables low-cost mass-production of compact, robust, multianalyte chemical sensor chips. The fabrication techniques which revolutionised electronics, making possible hugely complex microelectronic systems at very low cost, are enabling a similar transformation in optical devices. This renders optical circuits particularly well suited to mass-produced bio/chemical sensor arrays exploiting surface chemistry, optical cellsorters which discriminate on the basis of optical properties and for integration in microfluidic systems for advanced micro-cytometry. These planar optical chips are compatible with microfluidic systems for sample delivery and with optical fibre for solid-state connection to instrumentation.

\section{OPTICAL WAVEGUIDE BIOSENSORS}

In addition to low cost and the potential for a high level of integration, optimised monomode waveguides offer ultimate sensitivity, ultimate stability and the smallest sample volume, due to strong, localised evanescent interaction of light with liquid samples. They are ideal for excitation and interrogation of molecules at surfaces, being inherently surface sensitive devices [1].

Several highly sensitive and specific waveguide biosensors have been successfully demonstrated, and examples of biosensors based on SPR and on fluorescence will be described. The waveguide SPR immunosensors, which demonstrated detection limits for pesticides below 0.05 ppb [2], employed phase-matched excitation of propagating plasmons at a gold film surface from an underlying dielectric waveguide by tunnelling through the gold film. Biochemical specificity was achieved by modifying the gold surface with a molecule complementary to the target. Combined electrochemical and optical sensing, exploiting the gold SPR film as an electrode, has also been demonstrated [3].

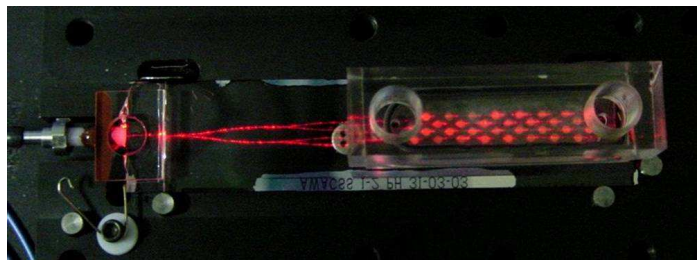

Fig. 1 Fluorescence biosensor array chip

For applications requiring lower detection limits, fluorescent labelling has been employed with fluorescence multisensor chips to yield a detection limit of 1ppt for oestrone, for example [4-6].

\section{OPTICAL TRAPPING AND PROPULSION}

Optical tweezers are well-established as a tool for non-contact, non-destructive handling of biological materials. Recently, interest has grown in optical manipulation at surfaces [7] as part of the toolbox of the "lab-on-a-chip". In particular, advances have been made in trapping and propulsion of dielectric microparticles and biological cells in the evanescent fields of optical waveguides [8], which may form part of a planar microsystem into which optical detection and spectroscopy of separated species could also be integrated.

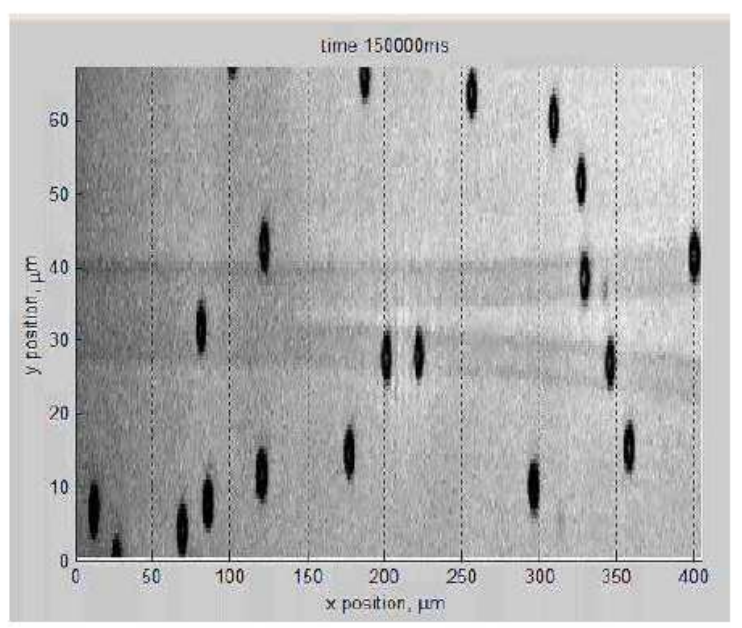

Fig. 2 Particle sorting on Y-branched waveguide 
Optical waveguides embedded in surfaces represent a powerful means of controlling the distribution of optical intensity and intensity gradient at such surfaces, for particle control, and recent results on the manipulation of biological cells will be described [9].

\section{INTEGRATED OPTOFLUIDICS}

Evanescent fields supported by optical waveguides are ideal probes for surface interactions. However, in applications where the volume of a liquid or of a micron-scale object such as a biological cell is to be interrogated, in-plane optofluidic approaches must be adopted [10,11]. Optical waveguide devices must be integrated with microfluidic channels to excite a defined volume in the fluid and to collect the scattered spectrum, in terms of angle or wavelength.

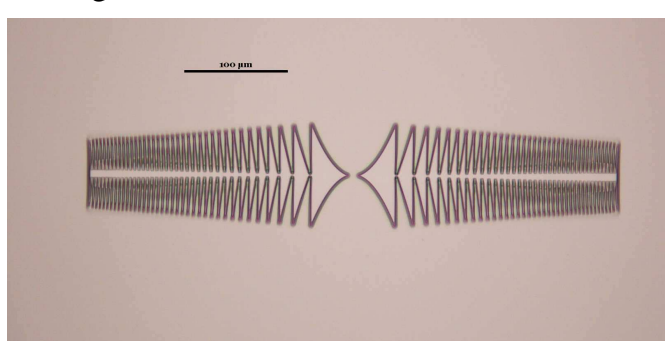

Fig. 3 Integrated kinoform lens

A key example function is that of the in-plane lens, which can be used for trapping, scattering, fluorescence or Raman measurements. Kinoform lenses are promising candidates for in-plane lenses, due to their compactness and flexibility in design [12]. Advances in technologies incorporating integrated lenses for microcytometry will be described.

\section{CONCLUSIONS}

The lab-on-a-chip presents great benefits in terms of reagent and sample consumption, speed, precision, and automation of analysis, and thus cost and ease of use, resulting in growing adoption of microfluidic approaches for practical measurements, and the potential for their widespread use in society. Optical techniques are ubiquitous in bio/chemical analysis and in the manipulation of biological cells, and optical waveguide devices show promise for on-chip integration with microfluidic systems for ultra-high performance, if low-cost approaches to fabrication and deployment can be achieved.

\section{REFERENCES}

[1] J.E. Midwinter "On the use of optical waveguide techniques for internal reflection spectroscopy" IEEE Journal of Quantum Electronics, 7, 339 ,1971.

[2] R.D.Harris, B.J.Luff, J.S.Wilkinson, J.Piehler, A.Brecht, G.Gauglitz \& R.A.Abuknesha, "Integrated Optical Surface Plasmon Resonance Immunoprobe for Simazine Detection", Biosensors \& Bioelectronics, 14, $377,1999$.

[3] A.K. Sheridan, P. Ngamukot, P.N. Bartlett \& J.S. Wilkinson, "Waveguide surface plasmon resonance sensing: electrochemical desorption of alkane thiol monolayers", Sensors \& Actuators B, 117, 253-260, 2006.

[4] P. Hua, J.P. Hole, J.S. Wilkinson, G. Proll, J. Tschmelak, G. Gauglitz, M.A. Jackson, R. Nudd, H.M.T. Griffith, R.A. Abuknesha, J. Kaiser \& P. Kraemmer, "Integrated optical fluorescence multisensor for water pollution", Optics Express, 13, 1124,2005 .

[5] J. Tschmelak et al., "Automated water analyser computer supported system (AWACSS) Part I: Project objectives, basic technology, immunoassay development, software design and networking", Biosensors \& Bioelectronics, 20, 1499-1508, 2005.

[6] J. Tschmelak et al., "Automated water analyser computer supported system (AWACSS) Part II: Intelligent, remote-controlled, cost-effective, online, water-monitoring measurement system", Biosensors \& Bioelectronics, 20, 1509-1519, 2005.

[7] K. Dholakia and P. Reece, "Optical micromanipulation takes hold", Nanotoday 1, 18-27, 2006.

[8] S. Kawata and T. Tani, "Optically-driven Mie particles in an evanescent field along a channeled waveguide", Optics Letters, 21, 1768-1770, 1996.

[9] B.S. Ahluwalia, O.G. Helles $\varnothing$, A.Z. Subramanian et al., "Integrated platform based on high refractive index contrast waveguide for optical guiding and sorting", Proc. Photonics West, San Francisco, CA USA, Jan. 23-28, 2010, 76130R. [10] T.N. Buican, M.J. Smyth, H.A. Crissman, G.C. Salzman, C.C. Stewart, and J.C. Martin JC "Automated single-cell manipulation and sorting by light trapping”, Appl. Opt., 26, 5311-5316, 1987.

[11] K.B. Mogensen, N.J. Petersen, J. Hübner, and J.P. Kutter "Monolithic integration of optical waveguides for absorbance detection in microfabricated electrophoresis devices", Electrophoresis 22, 3930-3938, 2001.

[12] H.C.Hunt and J.S.Wilkinson, "Integrated lenses for microfluidic systems", Proc. 15th Microoptics Conference MOC'09, Odaiba, Japan, Oct. 25-28, 2009, J14. 